\title{
Hybrid System of Communication and Radio Determination Using Two Geostationary Satellites
}

\author{
Shingo Ohmori, Yasushi Matsumoto and Eihisa Morikawa \\ Kashima Space Research Center \\ Communications Research Laboratory \\ Ministry of Posts and Telecommunications \\ Kashima, Ibaraki 314, Japan \\ Masayoshi Wakao \\ R \& D Center for Radio Systems \\ Hirakawa, Chiyoda, Tokyo, Japan
}

\section{Abstract}

Communications Research Laboratory (CRL) of the Ministry of Posts and Telecommunications has developed a new hybrid satellite system which can provide both communications and positioning services in one system using two geostationary satellites. The distinctive feature is that location information can be provided by transmitting and receiving ranging signals over the same channel as communications through two geostationary satellites.

\section{INTRODUCTION}

Mobile satelitte services are classified into two categories, one being "communtcations", providing volce, message, data transmission and so on, and the other being radio determination which gives information about location. In the past, these two satellite systems have been developed and implemented independently. However, in recent years, new satelite systems, which function both for communication and radio determination, have been studied by various organizations around the world.

Communications Research Laboratory (CRL) of the Ministry of Posts and Telecommunications has developed a new hybrid satellite system which can provide both communications and positioning services in one system using two geostationary satellites. The distinctive feature is that location information can be provided by transmitting and receiving ranging signals over the same channel as communications through two geostationary satellites.

The two types of terminals for the hybrid system developed by CRL are as follows:

Type A: in which one channel is used for both communication and positioning. A PN code ( 1023 chip rate) is used as a ranging signal and is transmitted over a single channel by OQPSK modulation.
Type B: in which the entire frequency bandwidth of SCPC channels is used. Communication and ranging information at 5 kbps, is spread by a PN coda ( 255 chip rate) into a $1.2 \mathrm{MHz}$ bandwidth and is transmitted by BPSK modulation.

This paper gives an the outline of system and the preliminary results.

\section{EXPERIMENTAL SYSTEM}

The experimental system consists of two geostationary sateliltes, ETS-V ( 150 E) and INMARSAT $(180 \mathrm{E})$, a base earth station (Kashima Space Research Center) and moblie earth stations [3]. The frequencles between the satellites and mobiles are $1.6 / 1.5 \mathrm{GHz}$. In order to determine the position of a mobile precisely. ranging accuracy is an essential factor, which depends on the performance of the test equipment and the prediction accuracy of satellite orbits. In order to evaluate system performance, the following two ranglng systems will be evaluated in the experiments.

\section{(1) Two-way Ranging}

As shown in Fig.1, a ranging signal is transmitted from the base earth station to a mobile station through the ETS-V satellite. The mobile station transmits the signal, which is repeated on IF or base band signal levels, to the base earth station through the ETS-V and INMARSAT satellites. The base station measures the time delay of reception between the respective signals from each satellite. In this system, the base station can determine the position of mobiles, so the mobiles are not required to have any complex functions for position determination. However, with this method satellite capacity is not efficiently used, because the mobile has to access to two satellites simultaneously. 


\section{- TIME COMPARISONS MODE}

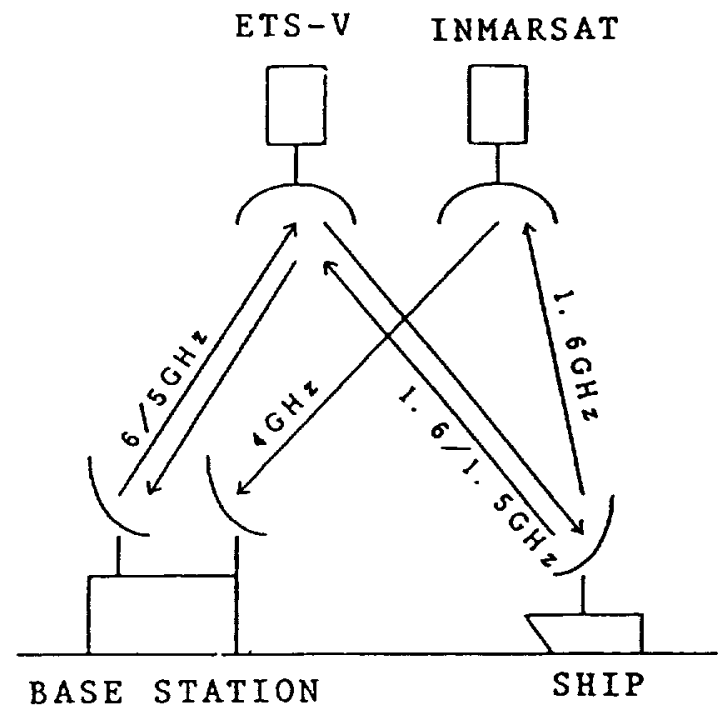

Fig.1 Two-way ranging.

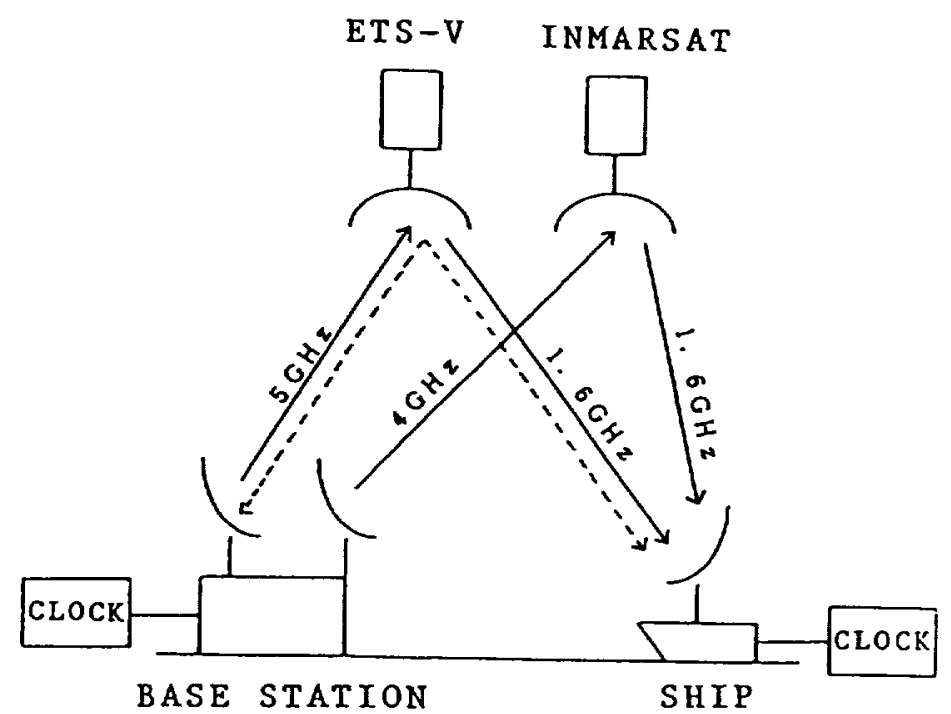

Fig.2 One-way ranging.

\section{(2) One-way Ranging}

As shown in Fig.2, the base station transmits the ranging signal, which is synchronized with a highly accurate clock, to a mobile station through the ETS-V and INMARSAT satellites. The mobile station measures the time delay with its clock, which is calibrated at appropriate intervals by the accurate clock through the ETS-V link. In this system, a mobile station can find its own position using the information of satellite orbits, which is provided by the base station through the satellite link.

Although, in this system, the mobile station has to have an additional function, whereby it can refer to the accurate time transmitted by the base station's master clock and adjust its own clock. Accordingly, this system can use a transponder more efficiently than the previous Two-way system.

\section{EQUIPMENT CONFIGURATION}

Two types of test equipment have been developed for the experiments with the hybrid satelilte communication system. The first one is called the SCPC method, which uses one communication channel in a single Channel Per Carrier (SCPC) access system for both communication and radio determination. The second one is called the SS method, which uses a spread spectrum technique to transmit and receive both the communication and ranging signals.

Both methods use digital modulation and demodulation techniques, and have ranging capabilities using a PN (pseudorandom noise) code. The Main characteristics of both methods are shown in Tablel.

Table 1. Method of modulation and parameters of ranging signals

\begin{tabular}{|c|c|c|}
\hline & $S C P C$ & $S S$ \\
\hline YETHOD OF MODULATION & $24 \mathrm{kbPS}$ OQPSK & $5 \mathrm{kbps}$ BPSK \\
\hline $\begin{array}{l}\text { SYMBOL RATE OF } \\
\text { ?Y SIGNALS }\end{array}$ & $12 \mathrm{kbps}$ & $\begin{array}{l}5 \mathrm{kbps} \times \\
1.275 \mathrm{Mbps}\end{array}$ \\
\hline ?: SIGNAL LENGTH & $1,000 \mathrm{chlps} .88 .33 \mathrm{msec}$ & $\begin{array}{l}500 \text { chips, } 100 \text { osec } * \\
255 \text { chips. } 0.2 \text { osec } * *\end{array}$ \\
\hline
\end{tabular}




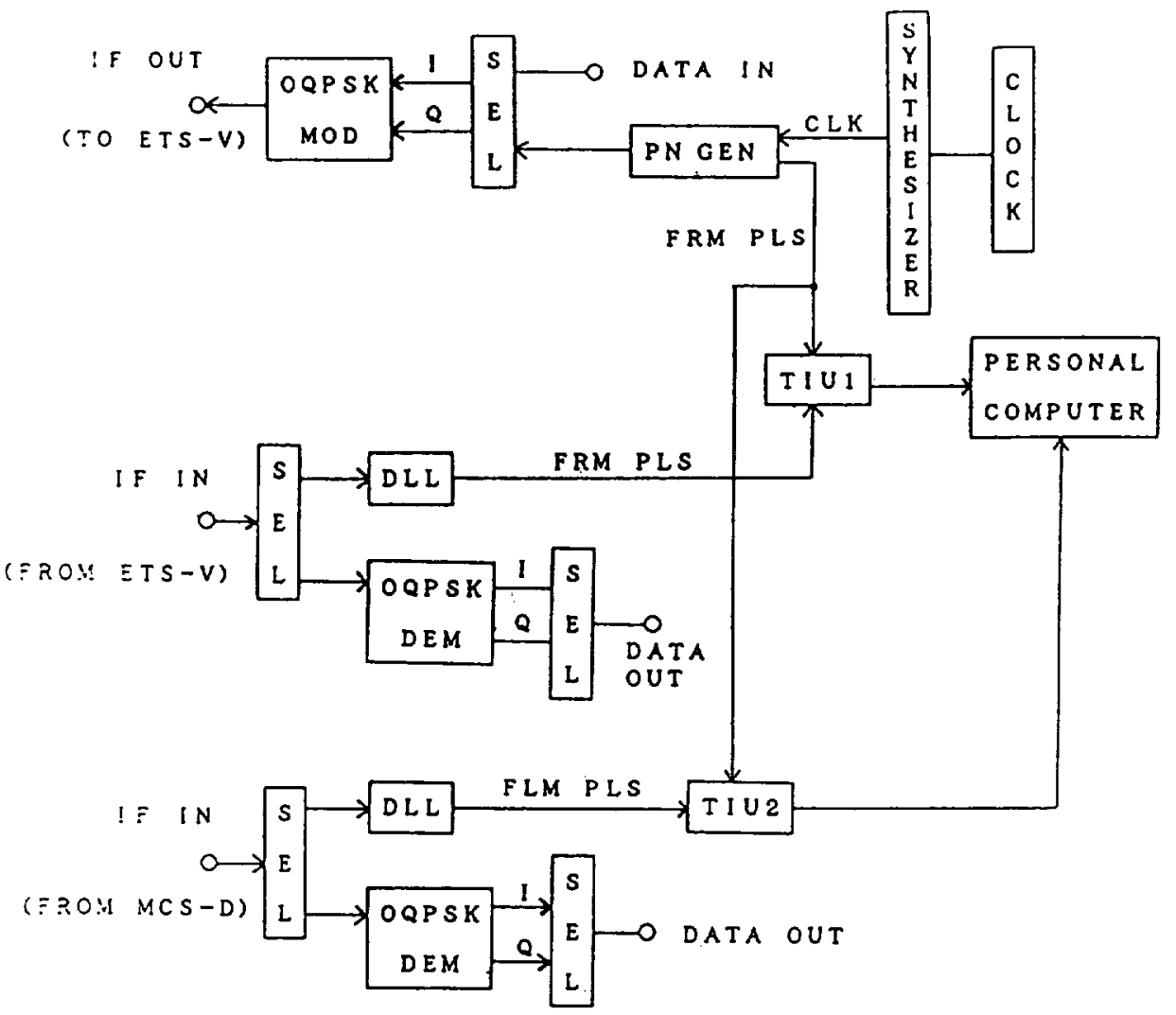

Fig.3 Base station block diagram. (SCPC ranging system)

(1) SCPC method

Fig.3 is a block dlagram of an SCPC terminal, which 1s connected to the RF section of the base station on IF signal levels. The PN code with 1023 chlps is used for the ranging signal, which modulates and demodulates the carrier through a $24 \mathrm{kbps}$-offset QPSK modulator (MOD) and demodulator(DEM). The $I$ and $Q$ channels of MOD/DEM are used for the communication signal and the ranging signal respectively. Synchronization of the recelved signal is locked by a Delay Lock Loop (DLL) with a Matched Pulse Detector (MPD). which consists of a digital correlation detector. The recelved PN signal is correlated with only 31-chip length of MPD, which allowing very fast acquisition of the initial carrier. The theoretical probabilities of mis-detection and falsedetection of received PN signals are shown in Fig.4. If an appropriate threshold level of detection is chosen, for example 25-bit, the probabilities of mis- and false-detection are expected to be as negligible as $10-7$ even in the case where Bit Error Rate (BER) is 10-2. A Time Interval Unit ( $T I U$ ) measures the time delay of the received ranging signal, which is transmitted by the PN generator unit of the base station. The data are processed by computer to decide the position of a mobile station, taking account of the data of satellite orbits.

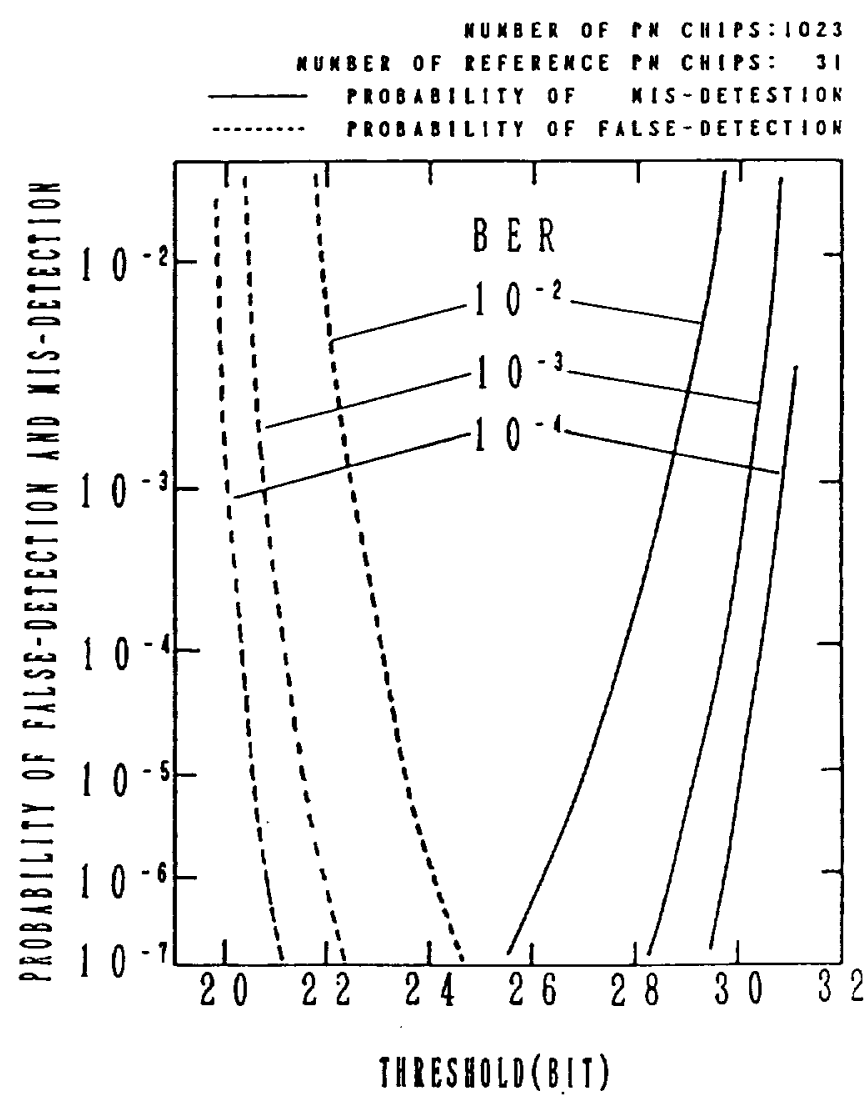

Fig.4 Probability of false- and mis-detection of matched pulse.

International Mobile Satellite Conference, Ottawa, 1990 


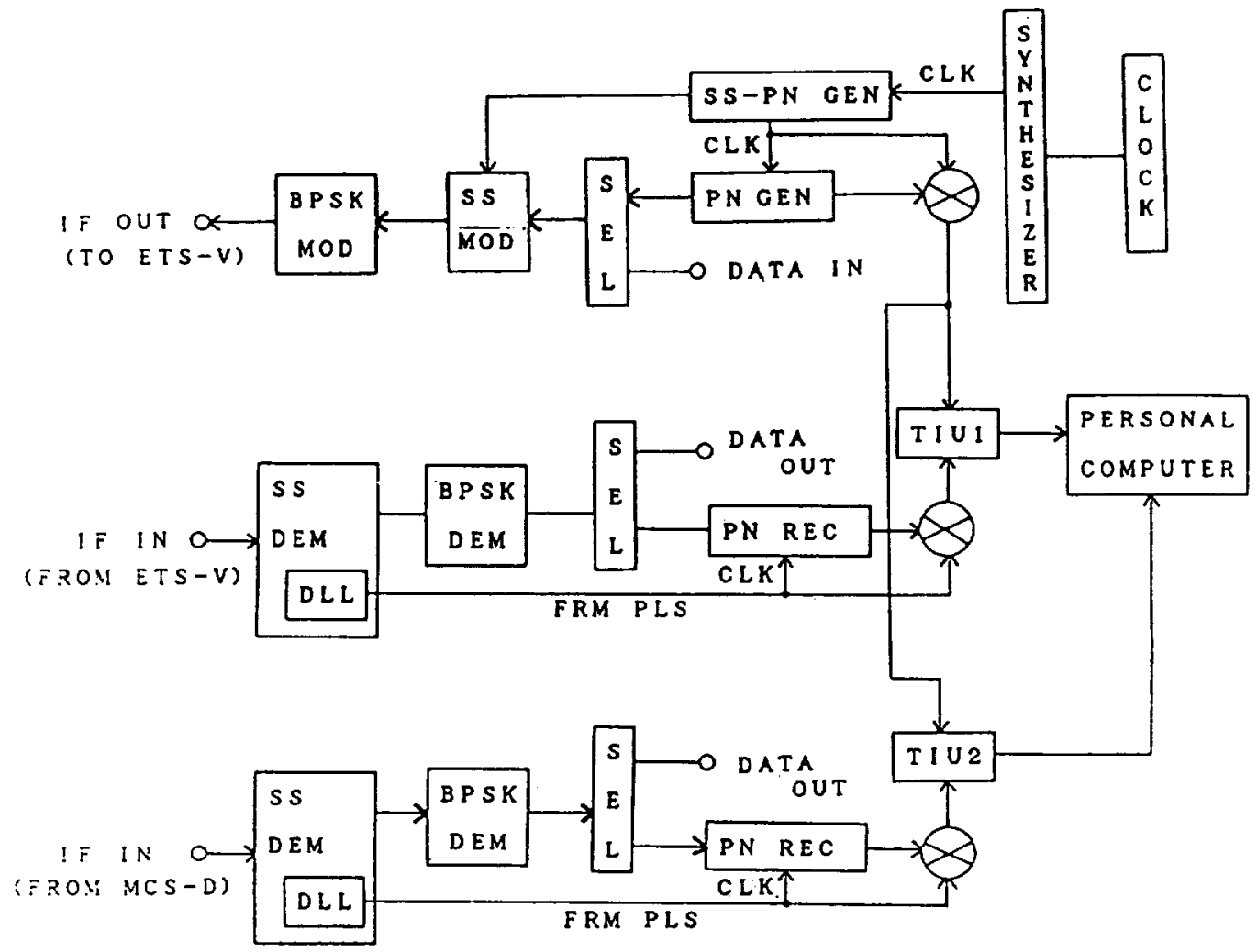

Fig. 5 Base station block diagram.

(sS ranging system)

\section{(2) SS method}

Fig.5 is a block diagram of the $S S$ test terminal, which is connected to the base station on IF signal levels. A base band signal of $5 \mathrm{kbps}$ is spread over a 1.2MHz frequency bandwidth by an SS modulator, and is transmitted through a BPSK modulator. The number of chips and length of frame of the PN code for the SS are 255 and $0.2 \mathrm{msec}$, respectively. This method has some ambiguity in deciding the time delay, because the time delay through a satelite link is greater than that of a frame length of the PN code. In order to eliminate the ambiguity, a 5kbps PN code is transmitted first, and when the initial acquisition is completed, voice and/or data can be transmitted instead of the 5kbps PN code through the same channel. At a receiving section, the DLL is used to synchronize with the recelved $P N$ code of $1.275 \mathrm{Mbps}$, which is used for spreading signals. The Time Interval Unit (TIU) measures the time delay of both PN signals, which are used for signal spreading and ambiguity elimination respectively. and a computer calculates the position of a mobile station with taking account of the information of sateliltes.

\section{Positioning Accuracy}

(a) Ranging Accuracy

The major ranging error is caused by the tracking error of the DLL in a receiving unit. The standard deviation of phase detection error is given by the following equation [5].

$$
\frac{\delta}{f}=\sqrt{\frac{B_{1} N_{a}}{2 P}}
$$

$\delta$ : standard deviation of phase detection error

fo: clock frequency of rangino signal

$B_{L}$ : equivalent noise bandwidth of $D L L$

Fig. 6 shows the theoretical results of phase detection error of the DLL in the SCPC and SS systems, calculated by the above equation.

The SCPC system has the advantage of a simple configuration, but the ranging accuracy is worse than that of the SS system because of its ranging signal's lower clock frequency. On the other hand, the SS system is affected by other communication carrier signals in the $s S$ band which reduce its channel quality $\mathrm{C} /$ No, the ratio of carrier to nolse density. Fig.? 


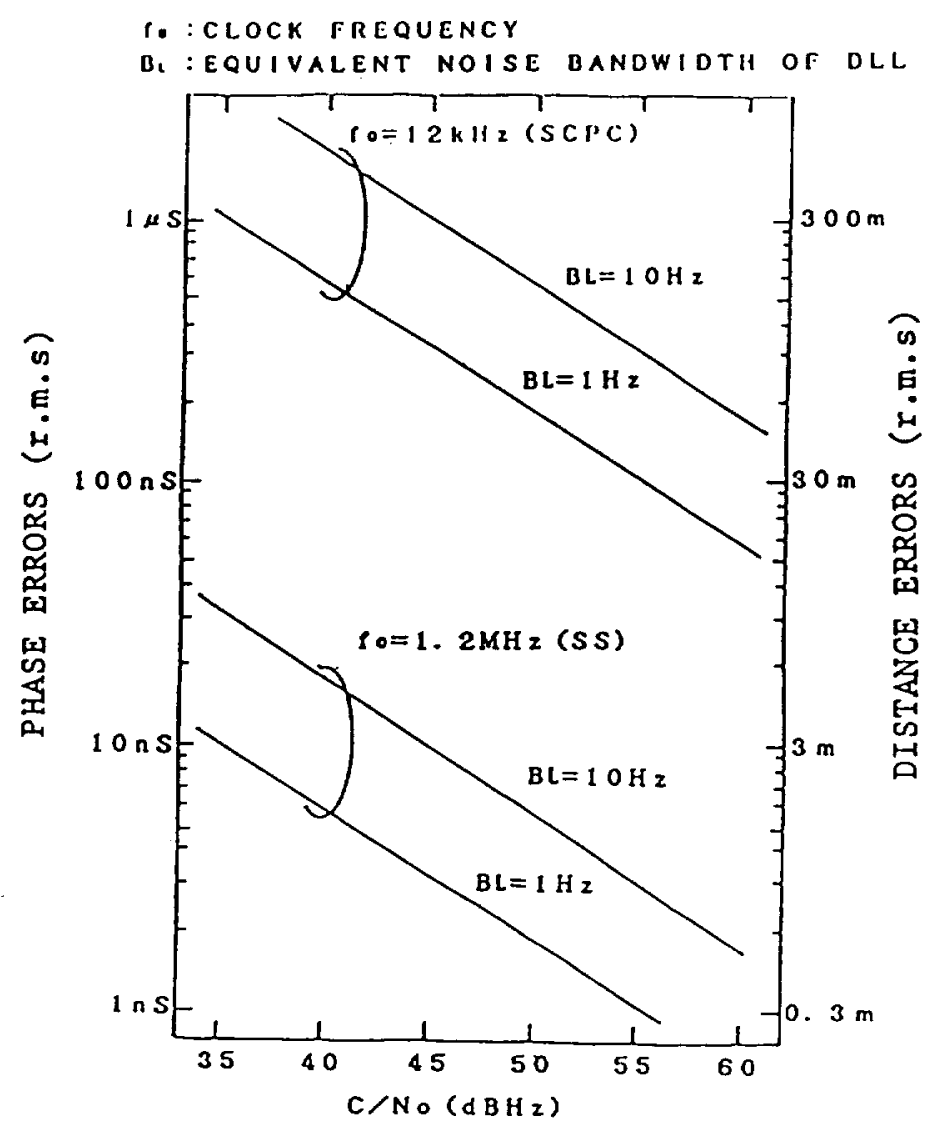

F1g.6 Recelved C/No versus phase errors of DLL.

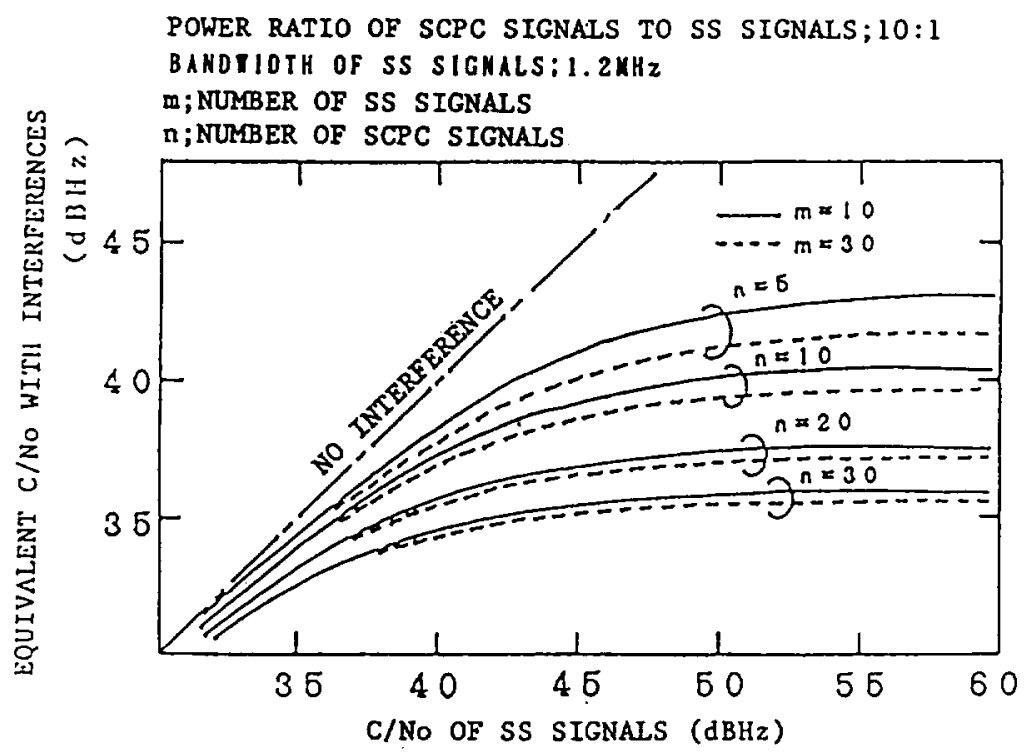

Fig. $7 \mathrm{C} /$ No for SS ranging system with interferences.

(Levels of SS and SCPC signals are equivalent.) 


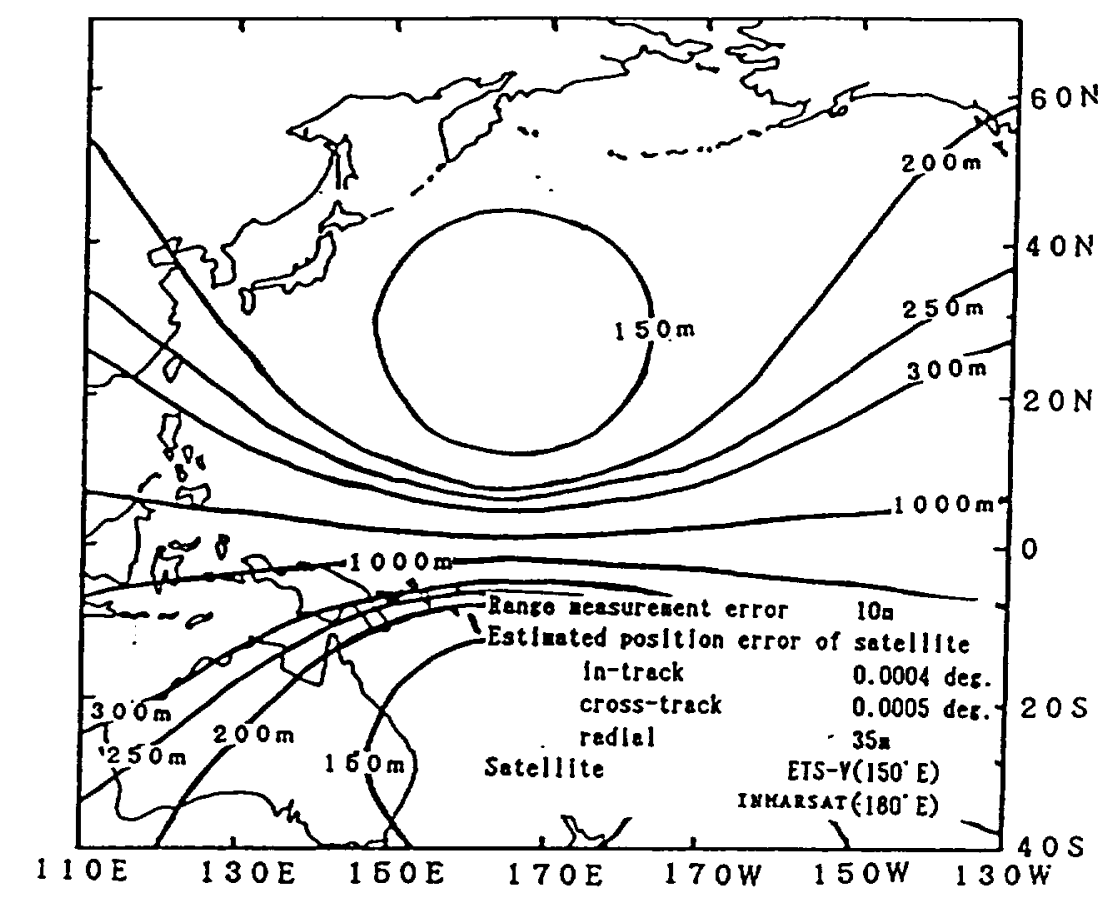

Fig.8 Accuracy of radio determination of this system.

shows the relation between the $\mathrm{C} /$ No and an equivalent $C /$ No with interference signals affecting the SS channel.

The Iink budget of the SCPC system predicts the channel quality of $\mathrm{C} /$ No to be about 47-58 dB, which will give a ranging accuracy of several tens of meters as shown in Fig.6. In the SS system, the expected $\mathrm{C} /$ No with interference signals is about 35-45 dB, which gives a ranging accuracy of about several meters.

\section{(b) Positioning Accuracy}

Positioning accuracy depends on both accuracy of ranging and satelite location information. Fig.8 shows an example of the positioning accuracy using ETS-V and INMARSAT sateliltes. The theoretical prediction is calculated under the assumption that ranging accuracy $1 \mathrm{~s} 10 \mathrm{~m}$, the amblguity of satellite location is 0.004 degrees in latitude, 0.0005 degrees in longltude and $35 \mathrm{~m}$ in radius. In low and middle latitude areas, a positioning accuracy of about several hundred meters is expected as shown in Fig.8. These figures are obtained without using any reference earth stations, but it is expected that greater accuracy can be obtained using reference stations which can eliminate many ranging blas exrors.

\section{CONCLUSION}

A hybrid satelilte system, which functions both for communication and radio determination, is proposed and the system configurations of two different methods are described. The proposed system can provide not only ordinary comrunications such as volce and data, but a radio determination service using a single communication channel. Radio determination and communication experiments using ETS-V and INMARSAT satellites are planned to start from the end of 1989. The experimental results axe expected to contribute to establishing new satellite services, which have dual missions of communications and radio determination.

\section{REFERENCES}

[1] G.K. O'Nai11;"The Geoster Radio Determination Satellite System". Telecommunication Journal, Vol.53, 1987.

[2] Y.Hase et al.; "Experimental Mobile sateliite System using ETS-V", IEEE Denshi Tokyo, No25, 1986.

[3] Miura et al.: "Code ranging system using ETS-V and INMARSAT system". Technical Report of IEICE of Japan, SAT8810,1988 .

[4] W.G111;"A Comparison of Binary DelayLock Tracking Implementations" , IEEE Trans.. Vol.AES-2, No.4, 1986. 